\title{
A report of acute overdosage of the anti-serotonergic drug pizotifen
}

\author{
A.P. Griffiths, N.D. Penn and H. Tindall \\ Department of Nuclear Medicine, General Infirmary at Leeds, Great George Street, Leeds LS1 3EX, UK.
}

\begin{abstract}
Summary: An acute overdosage of $30 \mathrm{mg}$ of pizotifen causing a pyrexial illness is reported. The clinical features suggest that the effects of overdosage are principally due to the anticholinergic activity of this drug. Resolution of symptoms occurred after 10 hours, without specific therapy.
\end{abstract}

\section{Introduction}

A recent review of the literature has revealed no published report of overdosage with pizotifen. We wish to report a case which recently presented to the accident and emergency department.

\section{Case report}

A 16 year old girl with a three year history of migraine and one year history of depression had been taking pizotifen $1 \mathrm{mg}$ three times daily for 2 months without adverse effects. On the day of admission she had taken $60 \times 0.5 \mathrm{mg}$ tablets of pizotifen (Sanomigran, Sandoz) four hours previously, but no other tablets. She was complaining of blurred vision and colicky abdominal pain. On examination she was drowsy, flushed, pyrexial at $38^{\circ} \mathrm{C}$, with dilated pupils, a sinus tachycardia of 120 beats/minute and blood pressure of $110 /$ $60 \mathrm{~mm} \mathrm{Hg}$. Gastric lavage failed to reveal any tablets. Twelve hours after admission she complained of feeling uncomfortably hot. Her skin was dry and her temperature had risen to $39^{\circ} \mathrm{C}$. Clinical examination revealed a sinus tachycardia but was otherwise unhelpful and investigations failed to reveal any cause for the pyrexia. Both the pyrexia and tachycardia persisted for approximately 10 hours before settling spontaneously (Figure 1).

\section{Discussion}

Pizotifen is a benzocycloheptathiophene which is structurally related to the tricyclic antidepressant drugs. It has strong antiserotonergic and antihistaminic effects but also possesses weak anticholinergic

Correspondence: A.P. Griffiths B.Sc., M.R.C.P.

Accepted: 11 August 1986 and antidepressant activity. Side effects of therapeutic doses comprise drowsiness and an increase in weight due to excessive appetite, and anticholinergic effects such as tachycardia, dry mouth and blurred vision. ${ }^{1}$ Pyrexia has not been reported as a complication of pizotifen taken in therapeutic dosage. Overdosage with histamine antagonists causes initial hyperpyrexia, central nervous system (CNS) stimulation and a clinical syndrome resembling atropine intoxication, attributable to their anticholinergic activity. When the overdose is large, CNS depression occurs with cardiovascular collapse and respiratory depression, and hypothermia may follow. ${ }^{2}$ Although pizotifen is marketed primarily as an inhibitor of serotonin, it does not seem possible to explain the pyrexia in this case as a consequence of its serotoninblocking activity. Serotonin is found in abundance in the hypothalamus and neighbouring structures where it is said to function as an inhibitory neurotransmitter.
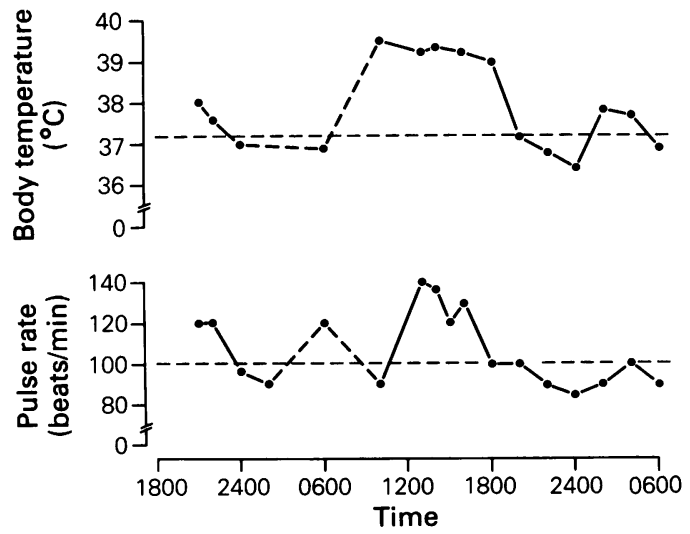

Figure 1 Time course of temperature and heart rate following overdose of pizotifen.

(C) The Fellowship of Postgraduate Medicine, 1987 
A recent review of the role of serotonin in hypothalamic temperature regulation concluded that an increase of serotoninergic activity resulted in an elevation of body temperature ${ }^{3}$ in which case hypothermia might be expected to follow an overdose of a serotonin inhibitor. It would seem more likely that the anticholinergic properties of pizotifen were responsible for the clinical manifestations of this overdose.

Intravenous physostigmine has been advocated for the treatment of overdosage with drugs possessing anticholinergic properties, principally for reversing central side effects such as delirium and coma. However, its short duration of action necessitates frequent administration (every 45 minutes or less) and

\section{References}

1. Speight, T.M. \& Avery, G.S. Pizotifen - a review of its pharmacological properties and its therapeutic efficacy in vascular headaches. Drugs, 1972, 3: 159-203.

2. Henry, J. \& Volans, G. ABC of Poisoning. Part 1. Drugs. BMJ Publications, London, 1984, p. 68.

3. Myers, R.D. Serotonin and thermoregulation: old and carries the risk, common to all analeptics, of $\cong$ precipitating convulsions. ${ }^{4}$ Since we are not able to $\frac{2}{\Phi}$ state the relative contributions of central and peri- $\stackrel{\mathbb{Q}}{\complement}$ pheral mechanisms to the production of pyrexia in $\stackrel{\hookrightarrow}{\hookrightarrow}$ pizotifen overdosage, we cannot predict what benefits $\stackrel{\vec{F}}{\stackrel{\vec{S}}{?}}$ might accrue from the use of physostigmine, or from the use of other anticholinesterase drugs which are not so effective in their penetration of the blood-brain barrier. Phenothiazines must not be used to reduce the temperature as they possess potent anti-muscarinic activity which will exacerbate the symptoms of overdosage. ${ }^{5}$ In the case reported above, symptomatic $\vec{\circ}$ treatment with tepid sponging and a cooling fan appeared to be sufficient.

new views. $J$ Physiol (Paris) 1981, 77: 505-513.

4. Vale, J.A. \& Meredith, T.J. Poisoning, Diagnosis and Treatment. Update Books, London, 1981, p. 93.

5. Gilman, A.G., Goodman, L.S., Rall, T.W. \& Murad, F. Goodman and Gilman's The Pharmacological Basis of Therapeutics, 7th Edn. Macmillan, London, 1985, p. 138. 